\title{
Single and multiple-spikes traveling wave solutions in integrate and fire neural networks Remus Oşan*
}

\author{
Address: Center for Systems Neurobiology, Departments of Pharmacology and Biomedical Engineering, Boston University, Boston, MA 02118, \\ USA \\ Email: Remus Oşan* - osan@bu.edu \\ * Corresponding author
}

from Sixteenth Annual Computational Neuroscience Meeting: CNS*2007

Toronto, Canada. 7-12 July 2007

Published: 6 July 2007

BMC Neuroscience 2007, 8(Suppl 2):P70 doi:10.1 I86/I47I-2202-8-S2-P70
We investigate the propagation of the traveling wave fronts in a one-dimensional integrate-and-fire network of synaptically coupled neurons for the case of one, two and multiple spike waves. We use an integro-differential equation characterizing the evolution of the firing times as a function of spatial position to determine the relationship between the speed of the propagating wave and its acceleration. We use the evolution equation to show that for a network of neurons with exponential synaptic connectivity and instantly rising, then exponentially decaying synapses, the evolution of the propagation is fully determined by the instantaneous speed of the traveling wave front. In this case the history of the firing map determines the initial speed of the transient propagation; the acceleration however depends only on the instantaneous speed, thus greatly simplifying the understanding of the network dynamics. This allows for a clear understanding of the conditions required for propagation failure, as well as of the mechanisms by which sustained transient propagation evolves towards the stable constant-speed traveling wave solution. Expanding the equation for the two and multiple wave cases yields further insight on the mechanisms by which sustained transient propagation evolves towards the stable constant-speed traveling wave solutions. In addition, we show that the wave speed and interspike intervals of the asymptotically stable state depend mainly on the interaction between a few successive wavefronts. It then follows that a unique asymptotical solution is selected from an infinite number of theoreti- cally possible solutions and that this solution is independent of the initial conditions in the neural network. 